\title{
Participatory Varietal Selection and Agronomic Evaluation of African Eggplant and Roselle Varieties in Mali
}

\author{
Meissa. Diouf \\ Chercheur en sélection végétale à TROPICASEM, Dakar, Sénégal \\ Mathieu Gueye \\ Chercheur en Botanique; Déprtement Botanique, et Géologie, à l'IFAN- \\ UCAD, Dakar, Sénégal \\ Papa Ibra Samb \\ Professeur titulaire au département de Biologie Végétale de l'UCAD, \\ Dakar, Fann, Sénégal
}

Doi: 10.19044/esj.2017.v13n30p327 URL:http://dx.doi.org/10.19044/esj.2017.v13n30p327

\begin{abstract}
African eggplant (Solanum aethiopicum L. and S. macrocarpon L.) is one of the most commonly consumed fruit vegetables in tropical Africa, in quantity and value probably the third after tomato and onion. Commercial production for supply to the cities is increasing. Roselle (Hibiscus sabdariffa L.) is another important vegetable crop in sub-Saharan Africa; its leaves, seed, and calyces are eaten. Roselle leaves and calyces are nutritious and rich in iron, $\beta$-carotene, and ascorbic acid. Although there is a market for African eggplant and Roselle, farmers have been slow to adopt new improved varieties due to use of traditional breeding method. To increase adoption rates of new varieties, we used a participatory varietal selection process to identify new varieties of both plants based on fruit yield, leaf, calyx traits, and consumers' preference. Fifteen lines and varieties of each species from several African countries were evaluated in Bamako, Mali during the 2008 hot rainy season. A randomized complete block design with three replications was used for the selection process. Twenty-two vegetable farmers and traders (or users) from Bamako rated the African eggplant and Roselle varieties based on visual characteristics (fruit color, fruit shape, number of fruits per plant, fruit size, number of secondary branches per plant, leaf size, leaf shape, leaf color, number of calyces per plant, calyx shape, and calyx color). Using a scale from 0 (least preferred) to 5 (most preferred), the varieties and the criteria were ranked, to identify the best variety and the most important criteria. In addition, agronomics data of African eggplant (number of fruits per plant, marketable yield, fruit weight,
\end{abstract}


fruits shape and fruit color) were collected. Agronomics data were also collected on Roselle calyces (number of calyces per plant, fresh calyces yield, dry calyces yield, dry matter and calyces color based on stem color) and leaves production (leaf length and width, economic yield, leaf shape and stem color). African eggplant line L10, varieties Soxna and Locale Gaya were highly rated for their visual appeal and have the potential to out-yield the local Malian variety. The fruit color and fruit shape, classified top two criteria through participatory selection criteria are important in the choice of users. For leafy entries of Roselle, leaf size was the most important trait. Samandah and line L28 were rated higher than the local check variety (Dah Rouge) for all traits. For calyx varieties of roselle, the number of calyces per plant was the most important trait. For leafy and calyx varieties, the agronomic evaluation show that yield is not a very important criterion in the choice of the users.

Keywords: Agronomic; calyx; criteria; fruit; leaf; Mali; preference; Senegal

\section{Introduction}

Sufficient levels of micronutrients and vitamins are essential for maintaining good health, especially for pregnant women and preschool children. Indigenous vegetables provide high levels of micronutrients, vitamins and fiber, and often are mainstays of diets in poor rural areas. Higher yielding, improved varieties could have a greater nutritional impact if they meet users' preferences for taste, color, shape, yield, nutritional, medicinal and economic values (AVRDC, 1998, Kamga et al., 2013, Njume et al., 2014 and Ray-Yu et al., 2013). African eggplant (Solanum aethiopicum L.) was domesticated from the wild species S. anguivi Lam. Both species are found throughout tropical Africa. The immature fruits of S. aethiopicum are used as a vegetable in stews, and sometimes eaten raw. The leaves and shoots are cooked; they are picked from the same plants that provide the fruit vegetable or from especially leafy cultivars. Fruits of bitter cultivars are used as medicine in many African countries. Small-scale growers account for at least $80 \%$ of the total production. Leaves of S. aethiopicum are especially important in southeastern Nigeria, Cameroon, and Uganda. It is a very popular leafy vegetable in Kampala markets. African eggplant cultivation for fruit and leaves is expanding in Tanzania because of its economic and nutritional value (Chadha and Mandinga, 2007). The leaves of the glabrous species (Solanum macrocarpon L.) are more nutritious than the fruit and are sometimes consumed in the same way as spinach in the southern part of Senegal (Seck, 1996 and Chippers, 2000). Roselle (Hibiscus sabdariffa L.), is another important vegetable crop in sub-Saharan Africa. Its leaves, seed, and calyces are eaten (Umerchuruba, 1997), and in many West 
African countries the dried calyces are prepared into a refreshing drink (Dignan et al., 1994). Roselle leaves are nutritious and have been reported to contain protein, Iron, $\beta$-carotene equivalent, thiamine, riboflavin, niacin, and ascorbic acid (Babalola et al., 2000 and Qi et al., 2005). Roselle seeds are ground into meal or are roasted and boiled as a coffee substitute (Scott, 2003). The international market for dried Roselle calyces is more than US\$120 million per year (Pasternack et al., 2004). Although there is a market for African eggplant and Roselle, farmers have been slow to adopt new improved varieties according to lack of use participatory variety selection method. In fact, there is a greater likelihood of farmers adopting a technology if they become involved early in project implementation (Rahman et al, 2015). To raise farmers' awareness and increase adoption rates of new varieties, the current research used a participatory varietal selection process (Getahun et al., 2016 and Ellsworth et al., 1992) to identify new varieties of both plants for the Sahelian region based on fruit yield, leaf, calyx traits, and users' preference. A similar study was conducted in Senegal on leafy roselle varieties (Diouf et al., 2007a). We would like to compare roselle preference varieties in Senegal and Mali on side and if relationship could be established between gronomic data and criteria users' preference.

\section{Materials and Methods}

Fifteen varieties of African eggplant from various African countries (Table 1) were evaluated at AVRDC's Samanko research station during the 2008 hot rainy season. Soil $\mathrm{pH}$ was between 4.5-6, the average rain was 807 $\mathrm{mm}$, the temperature between $21-41^{\circ} \mathrm{C}$ and the relative humidity between 72-87\%. Seed was sown on 7th July, and seedlings transplanted on 5th August. Before transplanting, basal fertilizer was applied, comprising $20 \mathrm{t} / \mathrm{ha}$ of compost and $250 \mathrm{~kg} / \mathrm{ha}$ mineral fertilizer NPK (10-10-20). The experimental design was a randomized complete block design (RCBD). Each treatment was replicated three times. There were two rows per plot, each $4 \mathrm{~m}$ long with $70 \mathrm{~cm}$ between the rows. Plants were spaced $50 \mathrm{~cm}$ apart in the rows. The total number of plots was 45 , including the check variety. Three side dressings of mineral fertilizer NPK (10-10-20) were applied: $200 \mathrm{~kg} / \mathrm{ha}$ at 40, 60 and 80 days after planting (Beniest, 1987).

Before sowing the fifteen varieties of Roselle from Ghana, Mali and Senegal (Table 3), basal fertilizer was applied, comprising $10 \mathrm{t} / \mathrm{ha}$ of compost and $300 \mathrm{~kg} / \mathrm{ha}$ mineral fertilizer NPK (10-10-20). The experimental design was a randomized complete block (RCBD). Each treatment was replicated three times. There were two rows per plot, each $4 \mathrm{~m}$ long, with 60 $\mathrm{cm}$ between the rows. Plants were spaced $40 \mathrm{~cm}$ apart in the rows. The total number of plots was 45, including the check variety. Two side dressings of mineral fertilizer NPK (10-10-20) were applied: $200 \mathrm{~kg} / \mathrm{ha}$ at 40 and 60 days 
after sowing (Beniest, 1987). Insects were controlled by applying deltametrine $(10 \mathrm{ml} \mathrm{/} 10 \mathrm{~L}$ of water) and fungi were controlled with Metalaxyl (400 ml / ha).

At 65 days after planting African eggplant, at 54 days after sowing leafy varieties and 106 days after sowing calyx varieties of Roselle, 22 vegetable farmers, with great experience, who are also traders (21 women; 1 man) from Bamako, a nearby urban area, were invited to participate in selecting varieties. The participants walked through the plots and discussed the traits of different lines and varieties. Before starting the scoring process, participants and researchers agreed that the most important traits were fruit shape, fruit color, fruit size, and number of fruits per plant, number of secondary branches per plant, leaf size, leaf shape, leaf color, number of calyces_per plant, calyx shape, and calyx color. A plastic container was placed in front of each line or variety for all the three replications of the trial. Each participant was given a cup filled with white bean seeds. Using a scale from 0 (least preferred) to 5 (most preferred), each participant dropped beans in the containers placed by each plot and this process is repeated for each trait. The total score was calculated for each criterion. In addition, agronomics data of African eggplant (number of fruits per plant, marketable yield, fruit weight, fruits shape and fruit color) for the fifteen entries were compared. The number of fruits per plant and the marketable yield were calculated based on 6 harvests at 35, 47, 49, 56, 64 and 72 days after transplantation (dat). The fruit weight was calculated using a sample of 5 fruits at the second harvest (47 dat) and third harvest (49 dat). Fruits shape and color were evaluated at the third harvest.

Agronomics data were also collected on calyces and leaves production of Roselle. For leafy varieties of Roselle it concerned leaf length and leaf width, economic yield, leaf shape and stem color. The agronomics data of calyx varieties concern the number of calices per plant, fresh calices yield, dry calices yield, dry matter and calices color based on stem color.

Using a sample of two plants at 54 days after sowing (das) leaf length and width, number of secondary branches, the economic yield, leaf shape and stem color were calculated. The fifth leaf from top was used for each plant in leaf length and width evaluation.

The number of calyces per plant, yield of fresh and dry calyces and calyces color were evaluated at 120 das using a sample of two plants. To evaluate calyx dry matter, a sample of fresh calyces $(20 \mathrm{~g})$ was collected on the two plant sampled in each variety. A sample of fresh calyx was dried during one week at room temperature $\left(24^{\circ} \mathrm{C}\right)$.

The score of users' preference traits obtained during participatory selection process and agronomics data collected were entered in Excel file 
and analyzed by STATISTIX. An Analysis of variance was performed followed by LSD All-Pairwise Comparisons test at 5\%.

\section{Results}

1. Ranking varieties of African eggplant according to agronomic data and participants' preference criteria

The evaluation of six agronomic parameters showed that varieties UG-AE-8 and UG AE-3 have the highest fruit number per plant. Varieties L10 followed by Locale Mali had the highest marketable yield and average fruit weight. These two last varieties were respectively, light green and dark green fruit colour with both ribbed and lightly flattened fruit shape (Table 1).

The L10 was ranked the best variety during the participatory selection (PS) process. It was followed by the Soxna variety and Locale Mali. The three varieties less preferred by users were respectively, UG-AE-8 $\left(13^{\text {th }}\right)$, UG-AE-3 $\left(14^{\text {th }}\right)$ and UG-AE-13 $\left(15^{\text {th }}\right)$ (Table 2$)$.

The ranking of the four criteria of users' preferences showed that fruit color was the most important trait (Table 4). It was followed by fruit shape, number of fruits per plant and average fruit size. The L10 had the highest score regarding the 4 criteria and confirmed the position of best variety among the 15 evaluated (Table 4). The two best varieties (L10 and Soxna) identified during PS process had light green fruit color, ribbed and lightly flattened fruit shape (Table 2).

Table 1 : Origin and agronomic performance of the 15 African eggplant varieties from research station

\begin{tabular}{|c|c|c|c|c|c|c|}
\hline Variety & Origine & FNPPL* & $\begin{array}{c}M Y^{* *} \\
(\mathrm{t} / \mathrm{ha})\end{array}$ & $\begin{array}{c}\mathrm{AFW} * * * \\
(\mathrm{~g})\end{array}$ & Fruit color & Fruit shape \\
\hline $\mathrm{AB} 2$ & $\begin{array}{l}\text { AVRDC- } \\
\text { Tanzanie }\end{array}$ & $04 \mathrm{c}$ & $8.380 \mathrm{ef}$ & $69.07 \mathrm{de}$ & whitish & $\begin{array}{l}\text { Smooth and } \\
\text { elongated }\end{array}$ \\
\hline RW-AE-1 & $\begin{array}{l}\text { Rwanda } \\
\text { (AVRDC- } \\
\text { Tanzanie) }\end{array}$ & $06 \mathrm{c}$ & 8.39 ef & $25.76 \mathrm{fg}$ & $\begin{array}{l}\text { Mixture Whitish } \\
\text { with dark green }\end{array}$ & Smooth and rounc \\
\hline Locale Mali & $\begin{array}{l}\text { AVRDC- } \\
\text { Mali }\end{array}$ & $04 \mathrm{c}$ & $22.98 \mathrm{ab}$ & $\begin{array}{c}145.51 \\
a b\end{array}$ & Dark green & $\begin{array}{c}\text { Ribbed and lightly } \\
\text { flattened }\end{array}$ \\
\hline UG-AE-10 & $\begin{array}{c}\text { Uganda } \\
\text { (AVRDC- } \\
\text { Tanzanie) }\end{array}$ & $05 \mathrm{c}$ & $4.80 \mathrm{f}$ & $21.19 \mathrm{fg}$ & Light green & $\begin{array}{c}\text { Smooth and } \\
\text { lightly elongated }\end{array}$ \\
\hline UG-AE-13 & $\begin{array}{l}\text { Uganda } \\
\text { (AVRDC- } \\
\text { Tanzanie) }\end{array}$ & $20 \mathrm{bc}$ & $3.53 \mathrm{f}$ & $14.90 \mathrm{fg}$ & $\begin{array}{l}\text { Mixture whitish with } \\
\text { green }\end{array}$ & Smooth and round \\
\hline
\end{tabular}




\begin{tabular}{|c|c|c|c|c|c|c|}
\hline TZ SMN3-10 & $\begin{array}{l}\text { AVRDC- } \\
\text { Tanzanie }\end{array}$ & $09 \mathrm{bc}$ & 8.30 ef & $20.43 \mathrm{fg}$ & whitish & $\begin{array}{l}\text { Smooth and } \\
\text { elongated }\end{array}$ \\
\hline $\begin{array}{l}\text { Manyire } \\
\text { Green }\end{array}$ & $\begin{array}{l}\text { AVRDC- } \\
\text { Tanzanie }\end{array}$ & $03 \mathrm{c}$ & $4.94 \mathrm{f}$ & 44.70 ef & dark green & $\begin{array}{c}\text { Lightly ribbed and } \\
\text { flattened }\end{array}$ \\
\hline UG-AE-3 & $\begin{array}{c}\text { Uganda } \\
\text { (AVRDC- } \\
\text { Tanzanie) }\end{array}$ & $45 \mathrm{a}$ & $5.05 \mathrm{f}$ & $2.34 \mathrm{~g}$ & dark green & Smooth and round \\
\hline UG-AE-20 & $\begin{array}{c}\text { Uganda } \\
\text { (AVRDC- } \\
\text { Tanzanie) }\end{array}$ & $26 \mathrm{~b}$ & 7.92 ef & $25.59 \mathrm{fg}$ & $\begin{array}{l}\text { Mixture Whitish } \\
\text { with dark green }\end{array}$ & $\begin{array}{c}\text { Smooth and } \\
\text { lightly elongated }\end{array}$ \\
\hline UVPP & $\begin{array}{l}\text { AVRDC- } \\
\text { Tanzanie }\end{array}$ & $03 \mathrm{c}$ & $13.24 \mathrm{de}$ & $94.86 \mathrm{~cd}$ & whitish & Smooth and round \\
\hline $\mathrm{N} 24$ & $\begin{array}{l}\text { AVRDC- } \\
\text { Tanzanie }\end{array}$ & $08 \mathrm{bc}$ & $18.64 \mathrm{bc}$ & 42.19 ef & whitish & $\begin{array}{l}\text { Smooth and } \\
\text { lightly round }\end{array}$ \\
\hline UG-AE-8 & & $57 \mathrm{a}$ & $7.61 \mathrm{f}$ & $26.38 \mathrm{fg}$ & Dark green & Smooth and round \\
\hline SOXNA & $\begin{array}{c}\text { ISRA- } \\
\text { CDH } \\
\text { Senegal }\end{array}$ & $03 \mathrm{c}$ & $\begin{array}{c}16.31 \\
\mathrm{~cd}\end{array}$ & $127.21 \mathrm{bc}$ & Light green & $\begin{array}{c}\text { Ribbed and lightly } \\
\text { flattened }\end{array}$ \\
\hline Locale Gaya & Niger & $02 \mathrm{c}$ & $\begin{array}{l}21.57 \\
\mathrm{abc}\end{array}$ & $171.03 \mathrm{a}$ & Dark green & Smooth and round \\
\hline L 10 & $\begin{array}{c}\text { ISRA- } \\
\text { CDH } \\
\text { Senegal }\end{array}$ & $04 \mathrm{c}$ & $24.04 \mathrm{a}$ & $97.46 \mathrm{~cd}$ & Light green & $\begin{array}{c}\text { Ribbed and lightly } \\
\text { flattened }\end{array}$ \\
\hline Grand Mean & & 13.3 & 11.7 & 61.9 & & \\
\hline $\begin{array}{l}\text { Standard } \\
\text { Deviation }\end{array}$ & & 18.86 & 7.6 & 55.13 & & \\
\hline
\end{tabular}

FNPPL* : fruit number per plant; $\mathrm{MY}^{* *}:$ Marketable Yield; $\mathrm{AFW}^{* * *}:$ Average Fruit Weight

Values in the same column with same letter(s) are not significantly different from each other according to LSD All-Pairwise Comparisons test at $5 \%$ probability.

Table 2: Ranking fifteen African eggplant varieties based on users' criteria

\begin{tabular}{|c|c|c|c|c|c|c|}
\hline Variety & NFPPL & Fruit size & Fruit color & Fruit shape & Total score & $\begin{array}{c}\text { Variety } \\
\text { rank }\end{array}$ \\
\hline L 10 & $102.00 \mathrm{a}$ & $106.00 \mathrm{a}$ & $92.33 \mathrm{a}$ & $104.33 \mathrm{a}$ & 404.7 & $1^{\text {st }}$ \\
\hline N24 & $86.67 \mathrm{ab}$ & $46.67 \mathrm{bcd}$ & $68.00 \mathrm{bc}$ & $51.00 \mathrm{de}$ & 252.3 & $5^{\text {th }}$ \\
\hline Locale Mali & $80.33 \mathrm{abc}$ & $74.00 \mathrm{~b}$ & $62.33 \mathrm{bcd}$ & $71.67 \mathrm{bc}$ & 288.3 & $3^{\text {th }}$ \\
\hline TZ MN3-10 & $75.00 \mathrm{bcd}$ & $32.33 \mathrm{cde}$ & $52.33 \mathrm{~d}$ & $37.67 \mathrm{ef}$ & 197.3 & $7^{\text {th }}$ \\
\hline
\end{tabular}




\begin{tabular}{|c|c|c|c|c|c|c|}
\hline UVPP & $70.33 \mathrm{bcd}$ & $60.00 \mathrm{bc}$ & $68.33 \mathrm{~b}$ & $57.67 \mathrm{~cd}$ & 256.3 & $4^{\text {th }}$ \\
\hline SOXNA & $61.67 \mathrm{~cd}$ & $55.67 \mathrm{bc}$ & $84.67 \mathrm{a}$ & $88.00 \mathrm{ab}$ & 290.0 & $2^{\text {nd }}$ \\
\hline Locale Gaya & $58.00 \mathrm{~d}$ & $61.00 \mathrm{bc}$ & $52.67 \mathrm{~cd}$ & $61.67 \mathrm{~cd}$ & 233.3 & $6^{\text {th }}$ \\
\hline AB2 & $30.67 \mathrm{e}$ & $20.67 \mathrm{det}$ & $49.67 \mathrm{~d}$ & $29.33 \mathrm{fg}$ & 130.3 & $8^{\text {th }}$ \\
\hline RW-AE-1 & $21.00 \mathrm{ef}$ & $17.67 \mathrm{def}$ & $22.00 \mathrm{e}$ & $23.00 \mathrm{fgh}$ & 83.7 & $9^{\text {th }}$ \\
\hline UG-AE-8 & $4.33 \mathrm{f}$ & $5.67 \mathrm{ef}$ & $12.00 \mathrm{e}$ & $12.33 \mathrm{hi}$ & 34.3 & $13^{\text {th }}$ \\
\hline UG-AE-10 & $2.33 \mathrm{f}$ & $3.67 \mathrm{ef}$ & $18.33 \mathrm{e}$ & $20.33 \mathrm{ghi}$ & 44.7 & $11^{\text {th }}$ \\
\hline UG-AE-3 & $2.33 \mathrm{f}$ & $1.00 \mathrm{f}$ & $11.00 \mathrm{e}$ & $6.67 \mathrm{hi}$ & 21.0 & $14^{\text {th }}$ \\
\hline UG-AE-13 & $2.00 \mathrm{f}$ & $0.00 \mathrm{f}$ & $11.00 \mathrm{e}$ & $6.00 \mathrm{i}$ & 19.0 & $15^{\text {th }}$ \\
\hline UG-AE-20 & $1.33 \mathrm{f}$ & $0.67 \mathrm{f}$ & $19.67 \mathrm{e}$ & $13.67 \mathrm{ghi}$ & 35.3 & $12^{\text {th }}$ \\
\hline Manyire Green & $1.00 \mathrm{f}$ & $10.00 \mathrm{ef}$ & $21.67 \mathrm{e}$ & $17.33 \mathrm{ghi}$ & 50.0 & $10^{\text {th }}$ \\
\hline Total score & 599 & 495 & 646 & 600.7 & & \\
\hline Criteria rank & $3^{\text {th }}$ & $4^{\text {th }}$ & $1^{\text {st }}$ & $2^{\text {nd }}$ & & \\
\hline
\end{tabular}

NFPPL* : number of fruits per plant

Values in the same column with same letter(s) are not significantly different from each other according to LSD All-Pairwise Comparisons test at $5 \%$ probability.

\section{Ranking leafy varieties of Roselle and identification of the most important traits}

The assessment of agronomic parameters confirmed the preference of the participants for the red type varieties than green types (Table 3). Regarding the economic yield, the red types were less productive than green. In fact yield was not the most important criterion. The leaf length, leaf width and the number of secondary branches did not show a statistically significant difference (Table 4). These three parameters were not greatly important in the preference of the participants.

Participants preferred varieties with red leaves (Table 4). The variety Samandah was ranked the best followed by L28. The first five varieties were red types while the green types were ranked at the last position, confirming the preference of users for the red types. Among all the criteria, the best was leaf size, followed by the number of secondary branches, the leaf shape and finally the leaf color (Table 4). The two varieties ranked best (Samandah and L28) had the highest score for leaf size criterion (Table 4).

Table 3: Agronomic performance of the 15 leafy varieties of Roselle from research station

\begin{tabular}{|c|c|c|c|c|c|c|c|}
\hline Variety & Origine & $\begin{array}{l}\mathrm{LL}^{*} \\
(\mathrm{~cm})\end{array}$ & $\begin{array}{r}\mathrm{LW}^{* *} \\
(\mathrm{~cm})\end{array}$ & NSBR $* * *$ & $\begin{array}{r}E Y * * * * \\
(\mathrm{~kg} / \mathrm{ha})\end{array}$ & Leaf shape & Stem color \\
\hline VIMTO & $\begin{array}{c}\text { ISRA- } \\
\text { CDH } \\
\text { Senegal }\end{array}$ & $10.57 \mathrm{ab}$ & $7.27 \mathrm{a}$ & $28.67 \mathrm{a}$ & $\begin{array}{c}120.00 \\
\text { bcd }\end{array}$ & $\begin{array}{c}\text { Light deep } 2 \\
\text { lobes }\end{array}$ & Red \\
\hline
\end{tabular}




\begin{tabular}{|c|c|c|c|c|c|c|c|}
\hline KOOR & $\begin{array}{c}\text { ISRA- } \\
\text { CDH } \\
\text { Senegal }\end{array}$ & $9.35 \mathrm{ab}$ & $6.05 \mathrm{ab}$ & $29.33 \mathrm{a}$ & $\begin{array}{c}100.00 \\
\mathrm{~cd}\end{array}$ & $\begin{array}{c}\text { Light deep } 2 \\
\text { lobes }\end{array}$ & Red \\
\hline $\begin{array}{c}\text { Marché de } \\
\text { Bazola }\end{array}$ & Mali & $8.62 \mathrm{ab}$ & $5.13 \mathrm{ab}$ & $28.00 \mathrm{ab}$ & $\begin{array}{c}162.23 \\
\text { abcd }\end{array}$ & $\begin{array}{c}\text { Light deep } 4 \\
\text { lobes }\end{array}$ & $\begin{array}{l}\text { Mixture red } \\
\text { and green }\end{array}$ \\
\hline ILAFIA & Mali & $8.28 \mathrm{~b}$ & $5.22 \mathrm{ab}$ & $26.67 \mathrm{ab}$ & $\begin{array}{c}189.17 \\
a b\end{array}$ & entire & green \\
\hline L24 & $\begin{array}{c}\text { ISRA- } \\
\text { CDH } \\
\text { Senegal }\end{array}$ & $8.70 \mathrm{ab}$ & $6.02 \mathrm{ab}$ & $23.00 \mathrm{~b}$ & $\begin{array}{c}179.73 \\
a b\end{array}$ & $\begin{array}{c}\text { Light deep } 4 \\
\text { lobes }\end{array}$ & Green \\
\hline $\mathrm{L} 22$ & $\begin{array}{c}\text { ISRA- } \\
\text { CDH } \\
\text { Senegal }\end{array}$ & $9.85 \mathrm{ab}$ & $6.20 \mathrm{ab}$ & $28.33 \mathrm{a}$ & $\begin{array}{c}187.09 \\
\mathrm{ab}\end{array}$ & $\begin{array}{c}\text { Very deep } 6 \\
\text { lobes }\end{array}$ & Green \\
\hline L7 & $\begin{array}{c}\text { ISRA- } \\
\text { CDH } \\
\text { Senegal }\end{array}$ & $9.27 \mathrm{ab}$ & $5.85 \mathrm{ab}$ & $28.00 \mathrm{ab}$ & $217.64 \mathrm{a}$ & $\begin{array}{c}\text { Light deep } 2 \\
\text { lobes }\end{array}$ & Green \\
\hline VERT CDH & $\begin{array}{c}\text { ISRA- } \\
\text { CDH } \\
\text { Senegal }\end{array}$ & $10.20 \mathrm{ab}$ & $6.02 \mathrm{ab}$ & $27.83 \mathrm{ab}$ & $\begin{array}{c}168.61 \\
\text { abcd }\end{array}$ & Deep 2 lobes & Green \\
\hline NOVORONGO & Ghana & $8.32 \mathrm{~b}$ & $5.18 \mathrm{ab}$ & $25.00 \mathrm{ab}$ & $215.14 \mathrm{a}$ & $\begin{array}{c}\text { Very deep } 2 \\
\text { lobes }\end{array}$ & Green \\
\hline MANDEKA & Mali & $10.90 \mathrm{a}$ & $5.95 \mathrm{ab}$ & $29.17 \mathrm{a}$ & $\begin{array}{c}174.73 \\
\mathrm{abc}\end{array}$ & $\begin{array}{c}\text { Light deep } 2 \\
\text { lobes }\end{array}$ & Green \\
\hline DAH BLANC & Mali & $9.43 \mathrm{ab}$ & $5.95 \mathrm{ab}$ & $26.00 \mathrm{ab}$ & $\begin{array}{c}132.64 \\
\text { bcd }\end{array}$ & $\begin{array}{c}\text { Light deep } 2 \\
\text { lobes }\end{array}$ & $\begin{array}{c}\text { Mixture } \\
\text { green and red }\end{array}$ \\
\hline SAMANDAH & $\begin{array}{l}\text { AVRDC } \\
\text { Bamako }\end{array}$ & $9.08 \mathrm{ab}$ & $5.42 \mathrm{ab}$ & $28.83 \mathrm{a}$ & $\begin{array}{c}126.11 \\
\text { bcd }\end{array}$ & entire & Red \\
\hline L 28 & $\begin{array}{c}\text { ISRA- } \\
\text { CDH } \\
\text { Senegal }\end{array}$ & $9.83 \mathrm{ab}$ & $5.90 \mathrm{ab}$ & $28.83 \mathrm{a}$ & $\begin{array}{c}134.86 \\
\text { bed }\end{array}$ & $\begin{array}{c}\text { Light deep } 2 \\
\text { lobes }\end{array}$ & Red \\
\hline $\begin{array}{c}\text { VERT } \\
\text { FATICK }\end{array}$ & $\begin{array}{c}\text { ISRA- } \\
\text { CDH } \\
\text { Senegal }\end{array}$ & $8.80 \mathrm{ab}$ & $5.05 \mathrm{ab}$ & $29.33 \mathrm{a}$ & $\begin{array}{c}140.00 \\
\text { bed }\end{array}$ & $\begin{array}{c}\text { Very deep } 4 \\
\text { lobes }\end{array}$ & Green \\
\hline DAH ROUGE & Mali & $8.08 \mathrm{~b}$ & $4.60 \mathrm{~b}$ & $27.33 \mathrm{ab}$ & $96.81 \mathrm{~d}$ & entire & $\begin{array}{c}\text { Mixture red } \\
\text { and green }\end{array}$ \\
\hline Grand Mean & & 9.3 & 5.7 & 27.6 & 156.3 & & \\
\hline Std deviation & & 1.5 & 1.3 & 3.1 & 53.2 & & \\
\hline
\end{tabular}

LL* : Leaf Length; LW** : Leaf Width; NSBR*** : number of secondary branches; EY**** : Economic Yield

Values in the same column with same letters are not significantly different from each other according to LSD All-Pairwise Comparisons test at $5 \%$ probability. 
Table 4: Ranking fifteen varieties of Roselle for leaf and calyx production based on users' criteria

\begin{tabular}{|c|c|c|c|c|c|c|}
\hline Variety & Leaf size & NSBR* & Leaf shape & Leaf color & Total score & $\begin{array}{c}\text { Variety } \\
\text { rank }\end{array}$ \\
\hline VIMTO & $86.33 \mathrm{abc}$ & $94.67 \mathrm{a}$ & $81.67 \mathrm{abcd}$ & $81.33 \mathrm{ab}$ & 257.67 & $4^{\text {th }}$ \\
\hline KOOR & $86 \mathrm{abc}$ & $91.33 \mathrm{a}$ & $85 \mathrm{abc}$ & $78 \mathrm{ab}$ & 254.33 & $5^{\text {th }}$ \\
\hline SAMANDAH & $100.33 \mathrm{a}$ & $89.67 \mathrm{a}$ & $101.33 \mathrm{a}$ & $100 \mathrm{a}$ & 291 & $1^{\text {st }}$ \\
\hline DAH BLANC & $79.67 \mathrm{bc}$ & $89.33 \mathrm{a}$ & $63.33 \mathrm{de}$ & $59 \mathrm{bc}$ & 211.66 & $7^{\text {th }}$ \\
\hline L28 & $100.33 \mathrm{a}$ & $86.67 \mathrm{ab}$ & $94 \mathrm{ab}$ & $90 \mathrm{a}$ & 270.67 & $2^{\text {nd }}$ \\
\hline DAH ROUGE & $90.33 \mathrm{abc}$ & $85.33 \mathrm{abc}$ & $88.33 \mathrm{abc}$ & $81.67 \mathrm{ab}$ & 255.33 & $3^{\text {th }}$ \\
\hline VERT CDH & $78 \mathrm{bc}$ & $81.33 \mathrm{abcd}$ & $61 \mathrm{e}$ & $58 \mathrm{bc}$ & 200.33 & $8^{\text {th }}$ \\
\hline Marché de Bazola & $45 \mathrm{~d}$ & $67.67 \mathrm{bcde}$ & $30 \mathrm{f}$ & $27.67 \mathrm{def}$ & 125.34 & $11^{\text {th }}$ \\
\hline ILAFIA & $75 \mathrm{c}$ & $65.33 \mathrm{cde}$ & $74 \mathrm{cde}$ & $42 \mathrm{cde}$ & 181.33 & $9^{\text {th }}$ \\
\hline L24 & $96 \mathrm{ab}$ & $64.67 \mathrm{de}$ & $79.33 \mathrm{bcde}$ & $93 \mathrm{a}$ & 237 & $6^{\text {th }}$ \\
\hline MANDEKA & $40 \mathrm{~d}$ & $56 \mathrm{ef}$ & $12.67 \mathrm{fg}$ & $9.67 \mathrm{f}$ & 78.34 & $13^{\text {th }}$ \\
\hline L7 & $76.33 \mathrm{bc}$ & $44 \mathrm{fg}$ & $76.33 \mathrm{bcde}$ & $54.67 \mathrm{bcd}$ & 175 & $10^{\text {th }}$ \\
\hline VERT FATICK & $25.33 \mathrm{de}$ & $43 \mathrm{fg}$ & $18.33 \mathrm{fg}$ & $13 \mathrm{f}$ & 74.33 & $14^{\text {th }}$ \\
\hline NOVORONGO & $38.67 \mathrm{de}$ & $33.67 \mathrm{gh}$ & $27.67 \mathrm{f}$ & $18.67 \mathrm{ef}$ & 80.01 & $12^{\text {th }}$ \\
\hline L22 & $8.67 \mathrm{e}$ & $19 \mathrm{~h}$ & $3.33 \mathrm{~g}$ & $2 \mathrm{f}$ & 24.33 & $15^{\text {th }}$ \\
\hline Total score criteria & 1025.99 & 1011.67 & 896.32 & 808.68 & & \\
\hline Criteria rank & $1^{\text {st }}$ & $2^{\text {nd }}$ & $3^{\text {th }}$ & $4^{\text {th }}$ & & \\
\hline NSBR & & & & & \\
\hline
\end{tabular}

NSBR* : number of secondary branches, Values in the same column with same letters are not significantly different from each other according to LSD All-Pairwise Comparisons test at $5 \%$ probability.

\section{Ranking calyx varieties and identification of the most important traits}

The assessment of agronomic parameters shows that the highest yielding varieties (fresh and dried calyx) were Samandah and L22 (Table 5). The two best ranked varieties during the participatory selection process had low yields. Although the number of calyces per plant was the first criterion, no correlation could be established with the yield of fresh and dried calyces. Variety ranked first by the participants was green type (L24) followed by red (L28) (Table 6). This shows that the color criterion is not very important. The shape and color are respectively, ranked second and third and no statistically significant difference was observed between the two varieties. The criterion number of calyces per plant ranked first remains the most discriminating in ranking the 15 calyx varieties of Roselle (Table 6).

Table 5 : Agronomic performance of the 15 calyx varieties of Roselle from research station

\begin{tabular}{|c|c|c|c|c|c|}
\hline Variety & NCPPL* & FCY** (t/ha) & $\begin{array}{c}\text { DCY**** } \\
(\mathrm{t} / \mathrm{ha})\end{array}$ & Dry matter $(\%)$ & $\begin{array}{c}\text { Calyx } \\
\text { color }\end{array}$ \\
\hline ILAFIA & $209 \mathrm{a}$ & $5.08 \mathrm{cdef}$ & $0.43 \mathrm{cde}$ & $7.98 \mathrm{cde}$ & Green \\
\hline DAH ROUGE & $189 \mathrm{a}$ & $7.48 \mathrm{abcd}$ & $1.08 \mathrm{ab}$ & $14.770 \mathrm{a}$ & Red \\
\hline
\end{tabular}




\begin{tabular}{|c|c|c|c|c|c|}
\hline Marché de Bazola & $177 \mathrm{ab}$ & $1.89 \mathrm{f}$ & $0.21 \mathrm{e}$ & $10.46 \mathrm{bc}$ & Green \\
\hline NOVORONGO & $146 \mathrm{abc}$ & $2.56 \mathrm{ef}$ & $0.27 \mathrm{de}$ & $10.76 \mathrm{bc}$ & Green \\
\hline MANDEKA & $144 \mathrm{abcd}$ & $7.79 \mathrm{abcd}$ & $0.70 \mathrm{bcde}$ & $9.23 \mathrm{bcde}$ & Green \\
\hline SAMANDAH & $129 \mathrm{abcde}$ & $10.43 \mathrm{a}$ & $1.51 \mathrm{a}$ & $14.42 \mathrm{a}$ & Red \\
\hline DAH BLANC & $107 \mathrm{bcdef}$ & $2.85 \mathrm{ef}$ & $0.19 \mathrm{e}$ & $6.29 \mathrm{de}$ & Green \\
\hline VERT CDH & $95 \mathrm{cdef}$ & $8.29 \mathrm{abc}$ & $0.79 \mathrm{bcd}$ & $9.58 \mathrm{bcd}$ & Green \\
\hline KOOR & $89 \mathrm{cdef}$ & $6.35 \mathrm{bcde}$ & $0.37 \mathrm{cde}$ & $5.71 \mathrm{e}$ & Red \\
\hline L28 & $88 \mathrm{cdef}$ & $6.53 \mathrm{abcde}$ & $0.59 \mathrm{bcde}$ & $9.11 \mathrm{bcde}$ & Red \\
\hline L22 & $70 \mathrm{cdef}$ & $9.36 \mathrm{ab}$ & $0.99 \mathrm{ab}$ & $9.12 \mathrm{bcde}$ & Green \\
\hline L24 & $70 \mathrm{cdef}$ & $3.81 \mathrm{def}$ & $0.31 \mathrm{de}$ & $8.09 \mathrm{cde}$ & Green \\
\hline L7 & $64 \mathrm{def}$ & $7.02 \mathrm{abcd}$ & $0.89 \mathrm{bc}$ & $12.39 \mathrm{ab}$ & Green \\
\hline VIMTO & $60 \mathrm{ef}$ & $5.51 \mathrm{bcdef}$ & $0.63 \mathrm{bcde}$ & $12.13 \mathrm{ab}$ & Dark red \\
\hline VERT FATICK & $47 \mathrm{f}$ & $5.39 \mathrm{bcdef}$ & $0.45 \mathrm{cde}$ & $8.40 \mathrm{cde}$ & Green \\
\hline Grand Mean & 112.06 & 6.02 & 0.63 & 9.89 & \\
\hline Standard deviation & 63.54 & 3.12 & 0.45 & $3 ; 37$ & \\
\hline
\end{tabular}

NCPPL* : number of calyces per plant; FCY** : fresh calyces yield; DCY*** : dry calyces yield

Values in the same column with same letters are not significantly different from each other according to LSD All-Pairwise Comparisons test at $5 \%$ probability

Table 6: Ranking calyces variety of Roselle based on users' criteria

\begin{tabular}{|c|c|c|c|c|c|}
\hline Variety & $\begin{array}{c}\text { Number of } \\
\text { calyces per } \\
\text { plant }\end{array}$ & $\begin{array}{c}\text { Calyces } \\
\text { shape }\end{array}$ & $\begin{array}{c}\text { Calyces } \\
\text { color }\end{array}$ & Total score & Variety rank \\
\hline L28 & $60 \mathrm{abc}$ & 92 & 77 & 229 & $2^{\text {nd }}$ \\
\hline L24 & $71 \mathrm{abc}$ & 91 & 71 & 233 & $1^{\text {st }}$ \\
\hline VIMTO & $37 \mathrm{bc}$ & 52 & 51 & 140 & $8^{\text {th }}$ \\
\hline DAH ROUGE & $45 \mathrm{abc}$ & 53 & 50 & 148 & $6^{\text {th }}$ \\
\hline Marché de Bazola & $28 \mathrm{c}$ & 47 & 48 & 123 & $12^{\text {th }}$ \\
\hline NOVORONGO & $27 \mathrm{c}$ & 49 & 45 & 121 & $13^{\text {th }}$ \\
\hline KOOR & $37 \mathrm{bc}$ & 49 & 44 & 130 & $10^{\text {th }}$ \\
\hline ILAFIA & $75 \mathrm{abc}$ & 55 & 42 & 172 & $3^{\text {th }}$ \\
\hline VERT CDH & $43 \mathrm{abc}$ & 58 & 40 & 141 & $7^{\text {th }}$ \\
\hline L7 & $71 \mathrm{abc}$ & 31 & 37 & 139 & $9^{\text {th }}$ \\
\hline SAMANDAH & $84 \mathrm{ab}$ & 37 & 37 & 158 & $4^{\text {th }}$ \\
\hline L22 & $59 \mathrm{abc}$ & 34 & 36 & 129 & $11^{\text {th }}$ \\
\hline VERT FATICK & $46 \mathrm{abc}$ & 36 & 35 & 117 & $14^{\text {th }}$ \\
\hline DAH BLANC & $94 \mathrm{a}$ & 30 & 25 & 149 & $5^{\text {th }}$ \\
\hline MANDEKA & $50 \mathrm{abc}$ & 3 & 2 & 55 & $15^{\text {th }}$ \\
\hline
\end{tabular}




\begin{tabular}{|c|c|c|c|l|l|}
\hline Total score of criteria & 827 & 714.12 & 638.1 & & \\
\hline Criteria rank & $1^{\text {st }}$ & $2^{\text {nd }}$ & $3^{\text {th }}$ & & \\
\hline
\end{tabular}

Values in the same column with same letters are not significantly different from each other according to LSD All-Pairwise Comparisons test at $5 \%$ probability.

\section{Discussion}

When conventional methods of breeding are used for African eggplant and Roselle, farmers have been slow to adopt new improved varieties. To increase adoption rates of new varieties, the researchers used a participatory varietal selection process to identify new varieties of both plants. In addition agronomics parameters were evaluated and their correlation with preference's criteria evaluated.

The process of ranking varieties of African eggplant according to agronomic data and participants' preference criteria show that the three varieties less preferred by users (UG-AE-8, UG-AE-3 and UG-AE-13) are produced in Uganda mainly for leaves consumption. In contrast, varieties L10, Soxna and Locale Mali, most preferred, are mainly grown in West Africa for fruit consumption. These results show that users' preference is highly related to locality and socio-economic aspects of people living in this area (Labrada, 2002).

Regarding the four criteria of users' preferences, fruit color and fruit shape are the two most important criteria in the choice of users during participatory selection process. These two criteria should be included as important trait during conventional breeding process.

Regarding leafy varieties of Roselle, among all the criteria, the best is leaf size, followed by the number of secondary branches, the leaf shape and finally the leaf color. The two varieties ranked best (Samandah and L28) have the highest score for leaf size criterion. The leaf size remains the most important trait (Diouf et al., 2007b). Malian participants preferred red type varieties, in contrast, Senegalese preferred the green types variety of Roselle (Diouf et al., 2006, Guèye and Diouf, 2006 and Van Rensburg et al., 2007), confirming that users' preference variation from one locality to another.

During the process of PS to rank calyx varieties and identify the most important traits, the yield is not a very important criterion. The criterion number of calyces per plant ranked first remains the most discriminating in ranking the 15 calyx varieties of Roselle. The calyx color is not very important in Mali, however the red calyx are more preferred in Senegal. 


\section{Conclusion}

Variety L10 is ranked the best variety during PS process; followed by Soxna and Locale Mali. Among the four criteria of users' preferences, fruit color is the most important. It is followed by fruit shape, number of fruits per plant and average fruit weight. The L10 has the highest total score throughout the 4 criteria and confirms the position as best variety among the 15 evaluated. Evaluation of six agronomic parameters show that the color and shape of the fruit, classified top two criteria through participatory selection criteria are important in the choice of users. These parameters should be included as important traits during selection process of new varieties with high potential of adoption.

Participants were able to identify the criteria of preference during PS process and used it for ranking the 15 Roselle varieties (leaf and calices types). The two best varieties of Roselle-leaves are Samandah and L28 and for calyces variety L24 and 28. Agronomic yield is not always an important criterion in ranking varieties during PS process. Participatory Selection process is an efficient tool in ranking varieties by users and help to improve rate of adoption of new varieties. The best varieties (eggplant, leafy and calyx varieties) could be included in advanced yield trials or multilocation evaluation trials. This experiment should be conducted at farmers' level, with separation between farmers and traders, to confirm or challenge results of this research. In addition, a further study is recommended to evaluate the most promising varieties with respect to cooking time and organoleptic taste.

\section{Acknowledgements}

All my acknowledgements to IPGRI (Bioversity International) for financial support

\section{References:}

1. AVRDC. (1998). Production for nutrition \& development». in Vegetables for Poverty Alleviation and Healthy Diets: A plan for 1998-2002. AVRDC (Eds). AVRDC, P.O. Box 42, Shanhua, Taiwan 741.

2. Babalola, S. O. Babalola, A. O. and Aworh, O. C. (2000). Compositional attributes of the calyces of Roselle (Hibiscus sabdarifa L.). J. Food Technol., Volume (6), 133-134.

3. Beniest, J. (1987). Guide pratique du maraîchage au Sénégal. CDHISRA, BP 3120. Dakar Sénégal.

4. Chadha, M. L. and Hasan Mndiga, H. (2007). African Eggplant From underutilized to a commercially profitable venture. ISHS Acta Horticulturae, Volume (752), 623. 
5. Dignan C. A. Burlingane, B. A. Arthur, J. M. Qinley, R. J. and Milligan, C. M. (1994). The Pacific Island Food Composition Tables. South Pacific Commission. Noumea. New Caledonia. New Zealand. pp 1-4.

6. Diouf, M. Guèye, M. Faye, B. Diémé, O. and Lô, C. (2007b). The commodity systems of four indigenous leafy vegetables in Senegal.. Water SA, Volume (33-3), 343-348.

7. Diouf, M. Diémé, O. Guèye, M. and Mbengue, N. B. (2006). Participatory selection of African leafyvegetables in Senegal». IPGRI Newsletter for Sub-Saharan Africa IPGRI-SSA. p. 9-10.

8. Diouf, M. Lô, C. Guèye, M. and Mbengue, N. B. (2007a). Participatory breeding of new genotypes selected in 4 species of african leafy vegetables (Hibiscus sabdariffa L., Amaranthus L. spp., Vigna unguiculata (L.) Walp. et Moringa oleifera Lam.)». AJFAND, Volume (7-3), 17.

9. Ellsworth, L. Diamé, F. Diop, S. and Thieba, D. (1992). Le Diagnostic Participatif ou "Participatory Rural Appraisal." Canda, CRDI Ottawa, pp. 110.

10. Getahun, A. Atnaf, M. Abady, S. Degu, T. and Dilnesaw, Z. (2016). Participatory variety selection of Soybean (Glycine max (L.) Merrill) varieties under rain fed condition of Pawe District, North-Western Ethiopia. Int J. of applied Science and Mathematics, Volume (3-I), 2394-2894.

11. Guèye, M. and Diouf, M. (2006). Traditional leafy vegetables in Senegal: diversity and medicinal uses. AJTCAM, Volume ( 4-4), 469-475.

12. Kamga, T. R. Kouamé, C. Atangana, A. R. and Chagomoka, T. (2013). Nutritional evaluation of five African indigenous vegetables. J. Hort. Res. Volume (21-1), 99-106.

13. Labrada, H. R. (2002). «Sélection végétale participative : cultiver le savoir. Point de vue. Spore, Volume (102), pp. 16.

14. Njume, C. Goduka, N. L. and George, G. (2014). Indigenous leafy vegetable (imifino, morogo) in South Africa : A rich unexplored source of nutrients and antioxidants. Afr. J. Biot. Volume (13-9), 1933-1942.

15. Pasternak, D. Nikiema, A. Dougbedji, F. Ndjeunga, J. K. S., Dan, G. A. and Abasse, T.. (2004). Agro management of Roselle, in The Sahelian Eco-Farm, ICRISAT (Ed.), Niger, Niamey (Ed.), p. 27.

16. Qi, Y. Chin, K. L. Malekian, F. Berhame, M. and Gager, I. (2005). Biological characteristics, nutritional and medicinal value of Roselle (Hibiscus sabdariffa). in Circular-Urban Forestry Natural Resources \& Environment. Volume (604). 
17. Rahman, R. A. Thant, A. A. Win, M. Tun, M. S., Moet Moet, P. Thu, A. M. Win, K. T. Myint, T. Tuntun, Y. Labios, R. V. Casimero, M. C. Gregorio, G. B. Johnson, D. E. Singleton, G. R. and Singh, R. K. (2015).Participatory varietal selection (PVS) a "Bottom up" breeding approach helps rice farmers in the Ayeyarwady Delta Myanman. SABRAO Journal of breeding and genetics, Volume (47-3), 299-314.

18. Ray-Yu, Yang and Chris, Ojiewo. (2013). African Nightshades and African Eggplants: Taxonomy, Crop Management, Utilization, and Phytonutrients. African Natural Plant Products Volume II: Discoveries and Challenges in Chemistry, Health, and Nutrition, pp.137-165, DOI:10.1021/bk-2013-1127.ch011.

19. Schippers, R. R. (2000). African Indigenous Vegetables: An Overview of the Cultivated Species. Natural Resources Institute. Chatham UK.

20. Scott, D. A. (2003). Red Sorrel, Hibiscus sabdariffa - The Other "Cranberry Plants \& Gardens News, Volume (18-2).

21. Seck, A. (1996). Conservation and utilization of germplasm of traditional African vegetables in Senegal.

22. TROPICASEM. Dakar. BP 9999, Senegal, 6p.

23. Umerchuruba, C. J. (1997). An annotated list of plant diseases in Nigeria. Pen \& Paper Publication. Owerri.Nigeria.

24. Van Rensburg, W. S. J. Van Averbeke, W. Slabbert, R. Faber, M. Van Jaarsveld, P. Van Heerden, I. Wenhold, F. and Oelofse, A. (2007). African leafy vegetables in South Africa, Water SA, Volume (33-3), 318-326. 